Article

\title{
Solvability Issues of a Pseudo-Parabolic Fractional Order Equation with a Nonlinear Boundary Condition
}

\author{
Serik E. Aitzhanov ${ }^{1,2}$, Abdumauvlen S. Berdyshev ${ }^{3, *}$ and Kymbat S. Bekenayeva ${ }^{3}$ \\ 1 Department of Mathematics, Al-Farabi Kazakh National University, Almaty A15E3B6, Kazakhstan; \\ serik.aitzhanov@kaznu.kz \\ 2 Department of Mathematics and Mathematical Modeling, International University of Information \\ Technologies, Almaty A15M0F0, Kazakhstan \\ 3 Department of Mathematics and Mathematical Modeling, Abai Kazakh National Pedagogical University, \\ Almaty 050010, Kazakhstan; kymbat.bekenayeva@mail.ru \\ * Correspondence: berdyshev@mail.ru
}

Citation: Aitzhanov, S.E.; Berdyshev, A.S.; Bekenayeva, K.S. Solvability Issues of a Pseudo-Parabolic Fractional Order Equation with a Nonlinear Boundary Condition. Fractal Fract. 2021, 5, 134. https:// doi.org/10.3390/ fractalfract5040134

Academic Editor: Adem Kilicman

Received: 12 August 2021

Accepted: 18 September 2021

Published: 23 September 2021

Publisher's Note: MDPI stays neutral with regard to jurisdictional claims in published maps and institutional affiliations.

Copyright: (c) 2021 by the authors. Licensee MDPI, Basel, Switzerland. This article is an open access article distributed under the terms and conditions of the Creative Commons Attribution (CC BY) license (https:/ / creativecommons.org/licenses/by/ $4.0 /)$.
Abstract: This paper is devoted to the fundamental problem of investigating the solvability of initialboundary value problems for a quasi-linear pseudo-parabolic equation of fractional order with a sufficiently smooth boundary. The difference between the studied problems is that the boundary conditions are set in the form of a nonlinear boundary condition with a fractional differentiation operator. The main result of this work is establishing the local or global solvability of stated problems, depending on the parameters of the equation. The Galerkin method is used to prove the existence of a quasi-linear pseudo-parabolic equation's weak solution in a bounded domain. Using Sobolev embedding theorems, a priori estimates of the solution are obtained. A priori estimates and the Rellich-Kondrashov theorem are used to prove the existence of the desired solutions to the considered boundary value problems. The uniqueness of the weak generalized solutions of the initial boundary value problems is proved on the basis of the obtained a priori estimates and the application of the generalized Gronwall lemma. The need to consider and study such initial boundary value problems for a quasi-linear pseudo-parabolic equation follows from practical requirements, such as solving fractional differential equations that simulate physical processes that occur during the study of liquid filtration processes, etc.

Keywords: pseudo-parabolic equation; the Caputo fractional derivative; Mittag-Leffler function; a priori estimates; Galerkin approximations; weak solution; global solvability; uniqueness of solution

\section{Introduction}

It is known that one of the most effective ways to study environmental processes using mathematical methods is their modeling in the form of differential equations. Many problems of natural science research have led to the study of various types of initialboundary values and direct and inverse problems for partial differential equations.

The interest in equations with mixed derivatives, which has increased recently for the pseudo-parabolic equation in particular, is explained by the needs of mechanics, other applied disciplines, and mathematical science itself. The solutions of many practically important problems arising in the study of liquid filtration processes in fractured porous media; the movement of underground water with a free surface in multilayer media; the transfer of moisture, heat, and salts in porous media; and so on are connected with the need to study boundary value problems for pseudo-parabolic equations of the third order [1-7]. For example, in [2], a one-dimensional initial-boundary value problem for a pseudo-parabolic equation with variable coefficients and a fractional Caputo derivative with respect to time variables is considered. A priori estimates are obtained, from which the uniqueness of the solution of the problem and its stability with respect to the initial 
data and the right part are proved. The convergence of the solutions of the associated difference problem to the solution of the differential problem is established.

Recently, fractional differential calculus has been widely used in the description of various mathematical models of physical processes [1,8-11]. In this regard, great attention is paid to partial differential equations of fractional order, which are a generalization of partial differential equations of integer order. At the same time, various statements are possible [6-8,12-15]. The paper [6] is devoted to the study of the solvability of boundary value problems with integral conditions for a class of quasi-linear pseudo-parabolic equations. The existence, uniqueness, and continuous dependence of a strong and weak generalized solution are proved. Applying the iterative process obtained for a linear problem, the existence, uniqueness, and continuous dependence of a weak generalized solution of a nonlinear problem are proved.

Boundary value problems for pseudo-parabolic equations and degenerate pseudoparabolic equations with a Caputo fractional derivative are studied in $[2,3,16]$. For loaded pseudo-parabolic equations of fractional order, we can note the work [17], where, with the help of the obtained priori estimates, the uniqueness of the solution and its stability with respect to the initial data and the right part are proved, as well as the convergence of the associated difference problem's solutions to the solution of the differential problem with speed $O\left(h^{2}+\tau\right)$, where $h$ and $\tau$ are steps in spatial and temporal variables.

In $[3,18,19]$, a non-local problem is studied for a pseudo-parabolic equation with fractional Caputo derivatives of the order $\sigma, 0<\sigma<1$ for time and spatial variables, where the fractional derivatives have the order $\alpha, \beta, \alpha>0, \beta>0$. Using the embedding theorem of S.L. Sobolev, the existence and uniqueness theorems of the problem are proved under certain restrictions on the order of the fractional derivatives $\alpha$ and $\beta$.

The works $[4-7,12-15,20-29]$ are devoted to the study of the solvability of various classes of problems for a pseudo-parabolic equation with a fractional derivative.

In this paper, the issues of local and global solvability of a problem with initial and nonlinear boundary conditions for a pseudo-parabolic equation with fractional Caputo derivatives are investigated. The existence and uniqueness theorems of the local and global generalized solutions of the problem are proved by the Galerkin approximation method.

The novelty of this work is the study of the issues of unique solvability of initial boundary value problems for a quasi-linear pseudo-parabolic fractional equation with nonlinear boundary conditions with Caputo fractional differentiation operators.

\section{Materials and Methods}

\subsection{Statement of the Problem}

We consider in the cylinder $Q_{T}=\left\{(x, t): x \in \Omega, \Omega \subset R^{n}, 0<t<T\right\}$ a quasi-linear pseudo-parabolic equation of fractional order:

$$
D_{0, t}^{\alpha}(u-\Delta u)-\Delta u+c(x, t) u=b(x, t)|u|^{p-2} u .
$$

where $\Omega \subset R^{n}, n \geq 3$ is a bounded domain, $\partial \Omega \in C^{2, \delta}, \delta \in(0,1)$, is smooth enough, $p$ and $\alpha$ are given positive constants, and $0<\alpha<1, c(x, t)$ and $b(x, t)$ are given functions, and $D_{0, t}^{\alpha} u(x, t)=\frac{1}{\Gamma(1-\alpha)} \int_{0}^{t} \frac{u_{s}(x, s)}{(t-s)^{\alpha}} d s$ is a fractional differentiation operator in a sense of Caputo [10].

Problem K. To find a solution $u(x, t)$ of Equation (1) that satisfies the initial condition:

$$
u(x, 0)=u_{0}(x), x \in \Omega,
$$

and non-linear boundary condition:

$$
D_{0, t}^{\alpha} \frac{\partial u}{\partial n}+\frac{\partial u}{\partial n}+\left.K(x, t)|u|^{\sigma-2} u\right|_{\Gamma}=0,
$$


where $\Gamma=\partial \Omega \times(0, T)$ and $\sigma$ is a positive constant.

We note that the solvability issues of the problem $\mathrm{K}$ in the case of $\alpha=1$ have been studied in [30]. For the integer parameter $\alpha$, problems with Dirichlet-Neumann boundary conditions have been investigated in [31,32].

We denote by $W^{\alpha}\left(Q_{T}\right), 0<\alpha<1$ Hilbert space with norm:

$$
\|u\|_{W^{\alpha}\left(Q_{T}\right)}^{2}=\|u\|_{L_{\infty}\left(0, T ; W_{2}^{1}(\Omega)\right)}^{2}+\left\|D_{0, t}^{\alpha} u\right\|_{L_{\infty}\left(0, T ; W_{2}^{1}(\Omega)\right)^{\prime}}^{2}
$$

where the norms of spaces are defined as follows:

$$
\begin{gathered}
\|u\|_{L_{p}(\Omega)} \equiv\|u\|_{p, \Omega}=\left(\int_{\Omega}|u|^{p} d x\right)^{\frac{1}{p}}, 1<p<\infty . \\
\|u\|_{L_{\infty}\left(0, T ; W_{2}^{1}(\Omega)\right)}^{2}=e \operatorname{sss} s u p_{t \in[0, T]}\|u\|_{W_{2}^{1}(\Omega)}^{2},\|u\|_{W_{p}^{l}(\Omega)}=\left(\sum_{k=0}^{l}\left\|D^{k} u\right\|_{p, \Omega}^{p}\right)^{\frac{1}{p}}, \\
D^{k} u=\frac{\partial^{|k|}}{\partial x_{1}^{k_{1}} \ldots x_{n}^{k_{n}}} ; k=\left(k_{1}, \ldots, k_{n}\right) \text { is multi-index, }|k|=k_{1}+\ldots+k_{n}, \\
D^{1} u \equiv D u \equiv \nabla u=\left(\frac{\partial u}{\partial x_{1}}, \ldots, \frac{\partial u}{\partial x_{n}}\right), D^{0} u=u .
\end{gathered}
$$

In what follows, we assume that the data of the problem satisfies the following conditions:

$$
\begin{gathered}
c(x, t) \geq c_{0}^{*}>0,0<b_{0} \leq b(x, t) \leq b_{1}, \forall(x, t) \in Q_{T}, \\
\left|D_{0, t}^{\alpha} b(x, t)\right| \leq b_{2}, 0<K_{0} \leq K(x, t) \leq K_{1}, \forall(x, t) \in Q_{T},
\end{gathered}
$$

Definition 1. A weak generalized solution of the problem $K(1)-(3)$ is a function $u(x, t) \in$ $W^{\alpha}\left(Q_{T}\right) \cap L_{\sigma}(\Gamma), 0<\alpha<1$ almost everywhere for $t \in[0, T]$ satisfying the following integral equality:

$$
\begin{aligned}
& \int_{\Omega}\left(D_{0, t}^{\alpha} u \cdot \omega+\sum_{i=1}^{n} D_{0, t}^{\alpha} \frac{\partial u}{\partial x_{i}} \cdot \frac{\partial \omega}{\partial x_{i}}+c(x, t) u \omega\right) d x \\
& +\int_{\Gamma} K(x, t)|u|^{\sigma-2} u \omega d \Gamma=\int_{\Omega} b(x, t)|u|^{p-2} u \omega d x,
\end{aligned}
$$

for all functions $\omega \in L_{2}\left(0, T ; W_{2}^{1}(\Omega)\right) \cap L_{\sigma}(\Gamma)$.

\subsection{Local Solvability of Problem K: Galerkin Approximations}

Let us consider the case where $p>2$ and let $u_{0}(x) \in W_{2}^{1}(\Omega)$.

In space $W_{2}^{1}(\Omega)$, we choose a system of functions $\left\{\Psi_{j}(x)\right\}$, forming a basis in this space. Due to the fact that the space $W_{2}^{1}(\Omega)$ is separable, such a system exists. The approximate solution of the problem to (1)-(3) is represented as follows:

$$
u_{m}(x, t)=\sum_{k=1}^{m} v_{m k}(t) \Psi_{k}(x),
$$

where $\left\{\Psi_{j}(x)\right\}$ is the orthogonal system of functions in $W_{2}^{1}(\Omega)$, and the coefficients $v_{m k}(t)$ are found from the system of equations:

$$
\begin{aligned}
\sum_{k=1}^{m} D_{0, t}^{\alpha} v_{m k}(t) \int_{\Omega}\left\{\Psi_{k} \Psi_{j} d x+\sum_{i=1}^{m} \frac{\partial \Psi_{k}}{\partial x_{i}} \frac{\partial \Psi_{j}}{\partial x_{i}}\right\} d x+\int_{\Omega} c(x, t) u_{m} \Psi_{j} d x+\int_{\Omega} \nabla u_{m} \nabla \Psi_{j} d x \\
\quad+\sum_{k=1}^{m} v_{m k} \int_{\Gamma} K(x, t)\left|u_{m}\right|^{\sigma-2} \Psi_{k} \Psi_{j} d \Gamma=\sum_{k=1}^{m} v_{m k} \int_{\Omega} b(x, t)\left|u_{m}\right|^{p-2} \Psi_{k} \Psi_{j} d x,
\end{aligned}
$$

with conditions

$$
u_{m 0}=u_{m}(0)=\sum_{k=1}^{m} v_{m k}(0) \Psi_{k}=\sum_{k=1}^{m} \alpha_{k} \Psi_{k}
$$


and

$$
u_{m 0} \rightarrow u_{0} \text { strongly in } W_{2}^{1}(\Omega) \text { at } m \rightarrow \infty,
$$

Let us introduce notations

$$
\begin{gathered}
\vec{v}_{m}=\left\{v_{1 m}(t), \ldots, v_{m m}(t)\right\}^{T}, \vec{\alpha} \equiv\left\{\alpha_{1}, \ldots, \alpha_{m}\right\}^{T}, a_{k j}=\int_{\Omega}\left[\Psi_{k} \Psi_{j}+\left(\nabla \Psi_{k} \nabla \Psi_{j}\right)\right] d x, \\
C_{k j}=\int_{\Omega} c(x, t) \Psi_{k} \Psi_{j} d x+\int_{\Omega} \nabla \Psi_{k} \nabla \Psi_{j} d x, \\
b_{k j}=-\int_{\Gamma} K(x, t)\left|u_{m}\right|^{\sigma-2} \Psi_{k} \Psi_{j} d \Gamma+\int_{\Omega} b(x, t)\left|u_{m}\right|^{p-2} \Psi_{k} \Psi_{j} d x, \\
A_{m} \equiv\left\{a_{j k}\right\}, \vec{C}_{m} \equiv\left\{C_{j k}\right\}, \vec{F}_{m}\left(\vec{v}_{m}\right) \equiv\left\{b_{j k}\left(\vec{v}_{m}\right)\right\} \vec{v}_{m} .
\end{gathered}
$$

Then, the system of Equation (7) with Condition (8) will take the following matrix form:

$$
A_{m} D_{0, t}^{\alpha} \vec{v}_{m}+\vec{C}_{m} \vec{v}_{m} \equiv \vec{F}_{m}\left(\vec{v}_{m}\right), \vec{v}_{m}(0)=\vec{\alpha}
$$

Below, we present several well-known lemmas necessary to establish some estimates.

Lemma 1. (see [23]) For any absolutely continuous on $[0, T]$ function $v(t)$, the following inequality holds:

$$
v(t) D_{0, t}^{\alpha} t^{2}(t) \geq \frac{1}{2} D_{0, t}^{\alpha} t^{2}(t), 0<\alpha<1
$$

Lemma 2. (see [25]) For any function $u \in W_{2}^{1}(\Omega)$, the inequality holds:

$$
\|u\|_{p, \Omega}^{p} \leq C_{1}\left(\|\nabla u\|_{2, \Omega}^{2}+\|u\|_{2, \Omega}^{2}\right)^{\frac{\theta p}{2}}\|u\|_{2, \Omega}^{(1-\theta) p} \leq C_{1}\left(\|\nabla u\|_{2, \Omega}^{2}+\|u\|_{2, \Omega}^{2}\right)^{\frac{\theta p}{2}+\frac{(1-\theta) p}{2}} \leq C_{1}\left(\|\nabla u\|_{2, \Omega}^{2}+\|u\|_{2, \Omega}^{2}\right)^{\frac{p}{2}},
$$

where

$$
C_{1}=\left(\frac{2(n-1)}{n-2}\right)^{\theta p}, \theta=\frac{(p-2) n}{2 p}, 0<\theta<1,2<p<\frac{2 n}{n-2}, n \geq 3 .
$$

Lemma 3. (see [25]) For any function $u \in W_{2}^{1}(\Omega)$, the inequality holds:

$$
\begin{gathered}
\|u\|_{q, \Gamma}^{q} \leq C_{1}\left(\|\nabla u\|_{2, \Omega}^{2}+\|u\|_{2, \Omega}^{2}\right)^{\frac{\theta p}{2}}\|u\|_{2, \Omega}^{(1-\theta) p} \leq C_{1}\left(\|\nabla u\|_{2, \Omega}^{2}+\|u\|_{2, \Omega}^{2}\right)^{\frac{\theta p}{2}+\frac{(1-\theta) p}{2}} \leq C_{1}\left(\|\nabla u\|_{2, \Omega}^{2}+\|u\|_{2, \Omega}^{2}\right)^{\frac{p}{2}}, \\
\text { where } \\
\Gamma=\partial \Omega \in C^{2, \delta}, \delta \in(0,1), C_{1}=\left(\frac{2(n-1)}{n-2}\right)^{\theta q}, \theta=\frac{(q-2) n+2}{2 q}, 0<\theta<1, \\
2<q<\frac{2(n-1)}{n-2}, n \geq 3 .
\end{gathered}
$$

We multiply both parts of (7) by $v_{m j}(t)$ and sum over $j=1, \ldots, m$; then, we obtain an equality:

$$
\begin{gathered}
\int_{\Omega}\left\{u_{m} D_{0, t}^{\alpha} u_{m} d x+\nabla u_{m} D_{0, t}^{\alpha} \nabla u_{m}\right\} d x+\int_{\Omega}\left|\nabla u_{m}\right|^{2} d x+\int_{\Omega} c(x, t)\left|u_{m}\right|^{2} d x \\
+\int_{\Gamma} K(x, t)\left|u_{m}\right|^{\sigma} d \Gamma=\int_{\Omega} b(x, t)\left|u_{m}\right|^{p} d x .
\end{gathered}
$$


By applying Lemma 1 and Lemma 2 to (10), and taking into account Condition (4), we obtain the following:

$$
\begin{gathered}
\frac{1}{2} D_{0, t}^{\alpha} \int_{\Omega}\left\{\left|u_{m}\right|^{2}+\left|\nabla u_{m}\right|^{2}\right\} d x+\int_{\Omega}\left|\nabla u_{m}\right|^{2} d x+c_{0}^{*} \int_{\Omega}\left|u_{m}\right|^{2} d x \\
+K_{0} \int_{\Gamma}\left|u_{m}\right|^{\sigma} d \Gamma \leq b_{1} C_{1}\left(\left\|u_{m}\right\|^{2}+\left\|\nabla u_{m}\right\|^{2}\right)^{\frac{p}{2}} .
\end{gathered}
$$

We write the resulting differential inequality as follows:

$$
D_{0, t}^{\alpha}\left(\left\|u_{m}\right\|^{2}+\left\|\nabla u_{m}\right\|^{2}\right)+C_{0}\left(\left\|u_{m}\right\|^{2}+\left\|\nabla u_{m}\right\|^{2}\right) \leq C_{2}\left(\|u\|^{2}+\left\|\nabla u_{m}\right\|^{2}\right)^{\frac{p}{2}},
$$

where $C_{0}=\min \left(1 ; c_{0}^{*}\right), C_{2}=2 C_{1} b_{1}$.

Defining $y(t)=\|u\|^{2}+\|\nabla u\|^{2}$, then we can represent (11) in the form:

$$
D_{0, t}^{\alpha} y(t)+C_{0} y(t) \leq C_{2}[y(t)]^{\frac{p}{2}} .
$$

From here, we obtain:

$$
y(t) \leq E_{\alpha, 1}\left(-C_{0} t^{\alpha}\right) y(0)+C_{2} \int_{0}^{t}(t-s)^{\alpha-1} E_{\alpha, \alpha}\left(-C_{2}(t-s)^{\alpha}\right)[y(s)]^{\frac{p}{2}} d s,
$$

where $E_{\alpha, \beta}(z)$ is the Mittag-Leffler function:

$$
E_{\alpha, \beta}(z)=\sum_{k=0}^{\infty} \frac{z^{k}}{\Gamma(\alpha k+\beta)} .
$$

Lemma 4. (see [9]) The following estimates are valid for the Mittag-Leffler function: if $0<\alpha<1$ and $\mu>0$, then there is a constant $M_{1}>0$ such that

$$
0<E_{\alpha, 1}\left(-\mu t^{\alpha}\right) \leq \frac{M_{1}}{1+\mu t^{\alpha}} \leq M_{1}, t>0 .
$$

if $0<\alpha<1$ and $\mu>0$, then there is a constant $M_{2}>0$, such that

$$
0<t^{\alpha-1} E_{\alpha, \alpha}\left(-\mu t^{\alpha}\right) \leq M_{2} t^{\alpha-1}, t>0 .
$$

From (12), taking into account (13) and (14), we have the following:

$$
y(t) \leq M_{1} y(0)+M_{2} C_{2} \int_{0}^{t}(t-s)^{\alpha-1}[y(s)]^{\frac{p}{2}} d s .
$$

Suppose that the following condition is satisfied:

$$
t<\left[\frac{2 \alpha}{(p-2) M_{2} C_{2}\left(M_{1} y(0)\right)^{\frac{p-2}{2}}}\right]^{\frac{1}{\alpha}}, 0 \leq t<T,
$$

then in (15), using the lemma of Gronwall-Bellman-Bihari [25], we obtain the inequality:

$$
y(t) \leq \frac{M_{1} y(0)}{\left[1-\frac{M_{2} C_{2}(p-2)}{2 \alpha}\left(M_{1} y(0)\right)^{\frac{p-2}{2}} t^{\alpha}\right]^{\frac{2}{p-2}}}
$$


or, considering the notation, we have:

$$
\begin{gathered}
\left\|u_{m}(x, t)\right\|_{2, \Omega}^{2}+\left\|\nabla u_{m}(x, t)\right\|_{2, \Omega}^{2} \\
\leq \frac{M_{1}\left(\left\|u_{m}(x, 0)\right\|_{2, \Omega}^{2}+\left\|\nabla u_{m}(x, 0)\right\|_{2, \Omega}^{2}\right)}{\left[1-(p-2) M_{1}^{\frac{p-2}{2}}\left(\left\|u_{m}(x, 0)\right\|_{2, \Omega}^{2}+\left\|\nabla u_{m}(x, 0)\right\|_{2, \Omega}^{2}\right)^{\frac{p-2}{2}} \frac{M_{2} C_{2}}{2 \alpha} t^{\alpha}\right]^{\frac{2}{p-2}}} .
\end{gathered}
$$

From this estimate, we can conclude that there is $T_{0}>0$, such that

$$
\left\|u_{m}\right\|_{2, \Omega}^{2}+\left\|\nabla u_{m}\right\|_{2, \Omega}^{2}+K_{0} \int_{\Gamma}\left|u_{m}\right|^{\sigma} d \Gamma \leq C_{3}, \text { for each } t \in[0, T], T<T_{0},
$$

where $C_{3}$ is a constant, independent of $m \in N$.

Now we multiply Equality (7) by $D_{0, t}^{\alpha} v_{m j}(t)$ and sum over $j=1, \ldots, m$. As a result, we obtain the following:

$$
\begin{gathered}
\left\|D_{0, t}^{\alpha} u_{m}\right\|_{2, \Omega}^{2}+\left\|\nabla D_{0, t}^{\alpha} u_{m}\right\|_{2, \Omega}^{2}= \\
-\int_{\Omega} \nabla u_{m} D_{0, t}^{\alpha} \nabla u_{m} d x-\int_{\Omega} c(x, t) u_{m} D_{0, t}^{\alpha} u_{m} d x \\
-\int_{\Gamma} K(x, t)\left|u_{m}\right|^{\sigma-2} u_{m} D_{0, t}^{\alpha} u_{m} d \Gamma+\int_{\Omega} b(x, t)\left|u_{m}\right|^{p-2} u_{m} D_{0, t}^{\alpha} u_{m} d x
\end{gathered}
$$

Applying the inequalities of Jung, Helder, and Lemma 3, we evaluate each term of the right part (17):

$$
\begin{gathered}
\left|-\int_{\Omega} \nabla u_{m} D_{0, t}^{\alpha} \nabla u_{m}\right| \leq \frac{1}{4}\left\|D_{0, t}^{\alpha} \nabla u_{m}\right\|^{2}+\left\|\nabla u_{m}\right\|^{2} . \\
\left|-\int_{\Omega} c(x, t) u_{m} D_{0, t}^{\alpha} u_{m} d x\right| \leq \frac{1}{2}\left\|D_{0, t}^{\alpha} u_{m}\right\|^{2}+\frac{c_{1}^{2}}{2}\left\|u_{m}\right\|^{2} . \\
\left.\left.\left|-\int_{\Gamma} K(x, t)\right| u_{m}\right|^{\sigma-2} u_{m} D_{0, t}^{\alpha} u_{m} d \Gamma\left|\leq K_{1} \int_{\Gamma}\right| D_{0, t}^{\alpha} u_{m}|| u_{m}\right|^{\sigma-1} d \Gamma \\
\leq K_{1}\left(\int_{\Gamma}\left|u_{m}\right|^{\frac{2(n-1)(\sigma-1)}{n}} d \Gamma\right)^{\frac{n}{2(n-1)}}\left(\int_{\Gamma}\left|D_{0, t}^{\alpha} u_{m}\right|^{\frac{2(n-1)}{n-2}} d \Gamma\right)^{\frac{n-2}{2(n-1)}} \\
\leq K_{1} C_{1}^{\frac{\sigma-1}{\sigma}} C_{3}^{\sigma-1}\left(\left\|D_{0, t}^{\alpha} u_{m}\right\|_{2, \Omega}^{2}+\left\|\nabla D_{0, t}^{\alpha} u_{m}\right\|_{2, \Omega}^{2}\right)^{\frac{1}{2}} \\
\leq K_{1}^{2} C_{1}^{2\left(\frac{2-1)}{\sigma}\right.} C_{3}^{2(\sigma-1)}+\frac{1}{4}\left(\left\|D_{0, t}^{\alpha} u_{m}\right\|_{2, \Omega}^{2}+\left\|\nabla D_{0, t}^{\alpha} u_{m}\right\|_{2, \Omega}^{2}\right),
\end{gathered}
$$

where $2<\sigma<\frac{2(n-1)}{n-2}, n \geq 3$.

$$
\begin{aligned}
& \left.\left.\left|\int_{\Omega} b(x, t)\right| u_{m}\right|^{p-2} u_{m} D_{0, t}^{\alpha} u_{m} d x\left|\leq b_{1} \int_{\Omega}\right| D_{0, t}^{\alpha} u_{m}|| u_{m}\right|^{p-1} d x \\
& \leq b_{1}\left(\int_{\Omega}|u|^{p} d x\right)^{\frac{1}{2}}\left(\int_{\Omega}\left|u_{m}\right|^{\frac{n(p-2)}{2}} d x\right)^{\frac{1}{n}}\left(\int_{\Omega}\left|D_{0, t}^{\alpha} u_{m}\right|^{\frac{2 n}{n-2}} d x\right)^{\frac{n-2}{2 n}} \\
& \leq b_{1} C_{1}^{\frac{p-1}{p}} C_{3}^{p-1}\left(\left\|D_{0, t}^{\alpha} u_{m}\right\|_{2, \Omega}^{2}+\left\|\nabla D_{0, t}^{\alpha} u_{m}\right\|_{2, \Omega}^{2}\right)^{\frac{1}{2}} \\
& \leq b_{1}^{2} C_{1}^{\frac{2(p-1)}{p}} C_{3}^{2(p-1)}+\frac{1}{4}\left(\left\|D_{0, t}^{\alpha} u_{m}\right\|_{2, \Omega}^{2}+\left\|\nabla D_{0, t}^{\alpha} u_{m}\right\|_{2, \Omega}^{2}\right),
\end{aligned}
$$

where $2<p<\frac{2 n}{n-2}, n \geq 3$.

Taking into account (16) and the above inequalities from Identity (17), we have a second a priori estimate:

$$
\left\|D_{0, t}^{\alpha} u_{m}\right\|_{2, \Omega}^{2}+\left\|\nabla D_{0, t}^{\alpha} u_{m}\right\|_{2, \Omega}^{2} \leq C_{4},
$$


for all $t \in[0, T], T<T_{0}$.

From these estimates, it follows that

$$
\begin{gathered}
u_{m} \text { is bounded in } L_{\infty}\left(0, T ; W_{2}^{1}(\Omega)\right) . \\
D_{0, t}^{\alpha} u_{m} \text { is bounded in } L_{\infty}\left(0, T ; W_{2}^{1}(\Omega)\right) . \\
K(x, t)\left|u_{m}\right|^{\sigma-2} \text { is bounded in } L_{\infty}\left(0, T ; L_{\sigma^{\prime}}(\Gamma)\right), \sigma^{\prime}=\frac{\sigma}{\sigma-1}>1 . \\
\left|u_{m}\right|^{p} \text { is bounded in } L_{\infty}\left(0, T ; W_{2}^{1}(\Omega)\right), 2<p<\frac{2 n}{n-2}, n \geq 3 . \\
\left|u_{m}\right|^{p-2} u_{m} \text { is bounded in } L_{\infty}\left(0, T ; L_{\frac{p}{p-1}}(\Omega)\right), 2<p<\frac{2 n}{n-2}, n \geq 3 .
\end{gathered}
$$

From (18) it follows that there exists subsequence $u_{m_{k}}$ of sequence $u_{m},{ }^{*}$ weakly converging to some element $L_{\infty}\left(0, T ; W_{2}^{1}(\Omega)\right)$, [31], i.e.:

$$
u_{m_{k}} \rightarrow u^{*} \text { weakly } L_{\infty}\left(0, T ; W_{2}^{1}(\Omega)\right) .
$$

Similarly, it follows from (18)-(22) that there is such a sequence $\left\{u_{m_{k}}\right\} \subset\left\{u_{m}\right\}$ where

$$
u_{m_{k}} \rightarrow u \text { weakly in } L_{2}\left(0, T ; W_{2}^{1}(\Omega)\right) .
$$

By virtue of the Rellich-Kondrashov theorem, the embedding $W_{2}^{1}\left(Q_{T}\right)$ is compact in $L_{2}\left(Q_{T}\right)$. This means that from the sequence $u_{m}$ we can choose the subsequence $u_{m_{k}}$ such that $u_{m_{k}} \rightarrow u$ in norm $L_{2}\left(Q_{T}\right)$, which means that it converges almost everywhere.

The above arguments allow us to pass to the limit at (7) when $m \rightarrow \infty$. First, we multiply both parts of Equality (7) by $d_{j}(t) \in C[0, T]$ and, summing both parts of the resulting identity on $\mathrm{j}=\overline{1, m}$, we have the following:

$$
\begin{aligned}
& \int_{\Omega}\left(D_{0, t}^{\alpha} u \cdot \mu+\sum_{i=1}^{m} D_{0, t}^{\alpha} \frac{\partial u}{\partial x_{i}} \cdot \frac{\partial \mu}{\partial x_{i}}+c(x, t) u \mu\right) d x \\
& +\int_{\Gamma} K(x, t)|u|^{\sigma-2} u \mu d \Gamma=\int_{\Omega} b(x, t)|u|^{p-2} u \mu d x,
\end{aligned}
$$

almost everywhere $t \in[0, T]$, where $\mu(x, t)=\sum_{j=1}^{m} d_{j}(t) \psi_{j}(x)$.

In (23) passing to the limit at $m \rightarrow \infty$, we obtain (5) for $\omega=\mu$. Since the set of all functions $\mu(x, t)$, where $\mu(x, t)=\sum_{j=1}^{m} d_{j}(t) \psi_{j}(x)$, is dense in $W^{\alpha}\left(Q_{T}\right) \cap L_{\sigma}(\Gamma)$, then that limit relation holds for all $\omega \in L_{2}\left(0, T ; W_{2}^{1}(\Omega)\right)$.

Thus, the following Theorem 1 is proved.

Theorem 1. Let Condition (4) be satisfied and

$$
2<p<\frac{2 n}{n-2}, 2<\sigma<\frac{2(n-1)}{n-2}, n \geq 3,
$$

then for any function $u_{0}(x) \in W_{2}^{1}(\Omega)$, almost everywhere $t \in(0, T), T<T_{0}$ where

$$
T_{0}=\left[\frac{2 \alpha}{2(p-2) b_{1} M_{2} M_{1}^{\frac{p-2}{2}}\left(\frac{2(n-1)}{n-2}\right)^{\frac{(p-2) n}{2}}\left(\|u(x, 0)\|_{2, \Omega}^{2}+\|\nabla u(x, 0)\|_{2, \Omega}^{2}\right)^{\frac{p-2}{2}}}\right]^{\frac{1}{\alpha}},
$$


There exists a weak generalized solution $u(x, t)$ of the problem $K(1)-(3)$, and the following inclusions take place:

$$
\begin{gathered}
u \in L_{\infty}\left(0, T ; W_{2}^{1}(\Omega)\right),\left|u_{m}\right|^{\frac{\sigma}{2}} \in L_{2}\left(\bar{Q}_{T}\right), \bar{Q}_{T}=(0, T) \times \Gamma, D_{0, t}^{\alpha} u \in L_{2}\left(0, T ; W_{2}^{1}(\Omega)\right), \\
|u|^{\sigma-2} u \in L_{\infty}\left(0, T ; L_{\sigma^{\prime}}(\Gamma)\right), \sigma^{\prime}=\frac{\sigma}{\sigma-1},|u|^{p} \in L_{\infty}\left(0, T ; W_{2}^{1}(\Omega)\right) .
\end{gathered}
$$

\subsection{Uniqueness of the Local Generalized Solution of the Problem K}

Theorem 2. Let $u_{0}(x) \in W_{2}^{1}(\Omega), 2<p<\frac{2 n}{n-2}, n \geq 3$, then generalized solution of the problem $K(1)-(3)$ on the interval $(0, T), T<T_{0}$ is unique.

Proof of Theorem 2. Suppose the problem K (1)-(3) has two solutions: $u_{1}(x, t)$ and $u_{2}(x, t)$. Then their difference $u(x, t)=u_{1}(x, t)-u_{2}(x, t)$ satisfies the condition $u(x, 0)=0$ and the identity

$$
\begin{gathered}
\int_{\Omega}\left(D_{0, t}^{\alpha} u \cdot \omega+\sum_{i=1}^{n} D_{0, t}^{\alpha} \frac{\partial u}{\partial x_{i}} \cdot \frac{\partial \omega}{\partial x_{i}}+c(x, t) u \omega\right) d x+\int_{\Gamma} K(x, t)\left(\left|u_{1}\right|^{\sigma-2} u_{1}-\left|u_{2}\right|^{\sigma-2} u_{2}\right) \omega d \Gamma= \\
\int_{\Omega} b(x, t)\left(\left|u_{1}\right|^{p-2} u_{1}-\left|u_{2}\right|^{p-2} u_{2}\right) \omega d x . \\
\text { By virtue of the fact that } \omega(x, t) \in L_{2}\left(0, T ; W_{2}^{1}(\Omega)\right) \text {, then we can take } u(x, t) \text { as } \omega(x, t) ; \\
\text { i.e., suppose } \omega(x, t)=u(x, t) \text {. Then, we have the following: } \\
\int_{\Omega}\left(D_{0, t}^{\alpha} u \cdot u+\sum_{i=1}^{n} D_{0, t}^{\alpha} \frac{\partial u}{\partial x_{i}} \cdot \frac{\partial u}{\partial x_{i}}+c(x, t)|u|^{2}\right) d x+\int_{\Gamma} K(x, t)|u|^{\sigma-2}\left(\left|u_{1}\right|^{\sigma-2} u_{1}-\left|u_{2}\right|^{\sigma-2} u_{2}\right) u d \Gamma= \\
\int_{\Omega} b(x, t)\left(\left|u_{1}\right|^{p-2} u_{1}-\left|u_{2}\right|^{p-2} u_{2}\right) u d x .
\end{gathered}
$$

We estimate the right-hand side of (25) by applying the following well-known algebraic inequalities:

$$
\begin{gathered}
\left.|| u_{1}\right|^{q} u_{1}-\left|u_{2}\right|^{q} u_{2}\left|\leq(q+1)\left(\left|u_{1}\right|^{q}+\left|u_{2}\right|^{q}\right)\right| u_{1}-u_{2} \mid, q>0, \\
\left|\left(\left|u_{1}\right| q u_{1}-\left|u_{2}\right| q u_{2}\right)\left(u_{1}-u_{2}\right)\right| \geq\left|u_{1}-u_{2}\right| q+2, q>0
\end{gathered}
$$

and Lemma 2. We then have:

$$
\begin{gathered}
\left|\int_{\Omega} b(x, t)\left(\left|u_{1}\right|^{p-2} u_{1}-\left|u_{2}\right|^{p-2} u_{2}\right) u d x\right| \leq b_{1}(p-1) \int_{\Omega}\left(\left|u_{1}\right|^{p-2}+\left|u_{2}\right|^{p-2}\right) u^{2} d x \\
\leq b_{1}(p-1)\left(\int_{\Omega}\left(\left|u_{1}\right|^{p-2}+\left|u_{2}\right|^{p-2}\right)^{2} u^{2} d x\right)^{\frac{1}{2}}\left(\int_{\Omega} u^{2} d x\right)^{\frac{1}{2}} \\
\leq b_{1}(p-1)\left(\int_{\Omega}\left(\left|u_{1}\right|^{p-2}+\left|u_{2}\right|^{p-2}\right)^{\frac{2 r}{r-2}} d x\right)^{\frac{r-2}{2 r}}\left(\int_{\Omega} u^{r} d x\right)^{\frac{1}{r}}\left(\int_{\Omega} u^{2} d x\right)^{\frac{1}{2}} \\
\leq b_{1}(p-1)\left(\left(\int_{\Omega}\left|u_{1}\right|^{\frac{2 r(p-2)}{r-2}} d x\right)^{\frac{r-2}{2 r}}+\left(\int_{\Omega}\left|u_{2}\right|^{\frac{2 r(p-2)}{r-2}} d x\right)^{\frac{r-2}{2 r}}\right) \times\left(\int_{\Omega} u^{r} d x\right)^{\frac{1}{r}}\left(\int_{\Omega} u^{2} d x\right)^{\frac{1}{2}},
\end{gathered}
$$

where $r=\frac{2 n}{n-2}, 2<p<\frac{2 n}{n-2}, n \geq 3$. Then, by the embedding theorem of Sobolev, $W_{2}^{1}(\Omega) \subset L_{r}(\Omega)$ and $W_{2}^{1}(\Omega) \subset L_{2 r(p-2) /(r-2)}(\Omega)$, taking into account the smoothness of solutions $u_{1}(x, t)$ and $u_{2}(x, t)$, we come to the following estimation:

$$
\left|\int_{\Omega} b(x, t)\left(\left|u_{1}\right|^{p-2} u_{1}-\left|u_{2}\right|^{p-2} u_{2}\right) u d x\right| \leq C_{5}\left(\|\nabla u\|_{2, \Omega}^{2}+\|u\|_{2, \Omega}^{2}\right) .
$$

From Estimate (27), taking into account Condition (4) and Lemma 1, as well as algebraic inequalities, we obtain:

$$
\begin{gathered}
D_{0, t}^{\alpha} \int_{\Omega}\left[|u|^{2}+|\nabla u|^{2}\right] d x+C_{0} \int_{\Omega}\left[|u|^{2}+|\nabla u|^{2}\right] d x+K_{0} \int_{\Gamma}|u|^{\sigma} d \Gamma \leq C_{5}\left(\|\nabla u\|_{2, \Omega}^{2}+\|u\|_{2, \Omega}^{2}\right), \\
\text { where } C_{0}=\min \left\{1 ; c_{0}^{*}\right\} .
\end{gathered}
$$


From Inequality (28) we obtain:

$$
D_{0, t}^{\alpha} \int_{\Omega}\left[|u|^{2}+|\nabla u|^{2}\right] d x \leq C_{5}\left(\|\nabla u\|_{2, \Omega}^{2}+\|u\|_{2, \Omega}^{2}\right)
$$

from which we have:

$$
\int_{\Omega}\left[|u|^{2}+|\nabla u|^{2}\right] d x \leq E_{\alpha, 1}\left(C_{5} t^{\alpha}\right) \int_{\Omega}\left[|u(x, 0)|^{2}+|\nabla u(x, 0)|^{2}\right] d x,
$$

that leads to $\int_{\Omega}\left(|\nabla u|^{2}+|u|^{2}\right) d x=0$ almost everywhere on the time interval $(0, T), T<T_{0}$, which represents the uniqueness of the generalized solution. Thus, Theorem 2 is proved.

2.4. The Existence of a Global Solution to the Problem K (1)-(3) for $1<p \leq 2$

Theorem 3. Let Condition (4) be satisfied and $1<p \leq 2,1<\sigma<\frac{2(n-1)}{n-2}, n \geq 3$. Then, for any $u_{0}(x) \in W_{2}^{1}(\Omega)$ on the interval $(0, T)$, there exists the generalized solution $u(x, t)$ of the Problem $K(1)-(3)$, and the following inclusions take place:

$$
u \in W^{\alpha}\left(Q_{T}\right) \cap L_{\sigma}(\Gamma) .
$$

Proof of Theorem 3. As in the proof of Theorem 1, multiplying both parts (7) by $v_{m j}(t)$ and summing over $\mathrm{j}=\overline{1, m}$, we obtain Relation (10).

The validity of the following lemma is given in [25].

Lemma 5. (see [25]) If $u \in W_{2}^{1}(\Omega), 1<q<2$, then the following inequality is true:

$$
\int_{\Omega}|u|^{q} d x \leq\left(\int_{\Omega}|u|^{2} d x\right)^{\frac{q}{2}}|\Omega|^{\frac{2-q}{2}} \leq c\left(1+\int_{\Omega}|u|^{2} d x+\int_{\Omega}|\nabla u|^{2} d x\right) .
$$

Applying Lemma 1 and the condition of Theorem 3, as well as Lemma 5, we obtain the inequality

$$
\begin{gathered}
D_{0, t}^{\alpha} \int_{\Omega}\left[\left|u_{m}\right|^{2}+\left|\nabla u_{m}\right|^{2}\right] d x+c_{0}^{*} \int_{\Omega}\left[\left|u_{m}\right|^{2}+\left|\nabla u_{m}\right|^{2}\right] d x+ \\
\int_{\Gamma} K(x, t)\left|u_{m}\right|^{\sigma} d \Gamma \leq c b_{1}\left(1+\left\|u_{m}\right\|_{2, \Omega}^{2}+\left\|u_{m}\right\|_{2, \Omega}^{2}\right) .
\end{gathered}
$$

Denoting $y(t) \equiv 1+\left\|u_{m}(x, t)\right\|_{2, \Omega}^{2}+\left\|\nabla u_{m}(x, t)\right\|_{2, \Omega}^{2}$, then the last inequality will be written as:

$$
D_{0, t}^{\alpha} y(t)+c_{0}^{*} y(t) \leq c b_{1} y(t)+c_{0}^{*} .
$$

From here, we obtain:

$$
\begin{gathered}
y(t) \leq E_{\alpha, 1}\left(-c_{0}^{*} t^{\alpha}\right) y(0)+c b_{1} \int_{0}^{t}(t-s)^{\alpha-1} E_{\alpha, \alpha}\left(-c_{0}^{*}(t-s)^{\alpha}\right) y(s) d s+ \\
\int_{0}^{t}(t-s)^{\alpha-1} E_{\alpha, \alpha}\left(-c_{0}^{*}(t-s)^{\alpha}\right) c_{0}^{*} d s,
\end{gathered}
$$

From Inequality (29), by virtue of (13) and (14), we have:

$$
y(t) \leq M_{1} y(0)+\frac{M_{2} c_{0}^{*} t^{\alpha}}{\alpha}+c b_{1} M_{2} \int_{0}^{t}(t-s)^{\alpha-1} y(s) d s .
$$

The proof of the following lemma is given in [33]. 
Lemma 6. (see [33]) Suppose $b \geq 0, \beta>0$ and $a(t)$ is a nonnegative function locally integrable on $0 \leq t \leq T$ with

$$
u(t) \leq a(t)+b \int_{0}^{t}(t-s)^{\beta-1} u(s) d s,
$$

on this interval, then

$$
u(t) \leq a(t)+\theta \int_{0}^{t} E_{\beta}^{\prime}(\theta(t-s)) a(s) d s, 0 \leq t \leq T,
$$

where

$$
\begin{gathered}
\theta=(b \Gamma(\beta))^{\frac{1}{\beta}}, E_{\beta}(z)=\sum_{n=0}^{\infty} \frac{z^{n \beta}}{\Gamma(n \beta+1)}, E_{\beta}^{\prime}(z)=\frac{d}{d z} E_{\beta}(z), \\
E_{\beta}^{\prime}(z) \rightarrow \frac{z^{\beta-1}}{\Gamma(\beta)} \text { as } z \rightarrow+0, E_{\beta}^{\prime}(z) \rightarrow \frac{e^{z}}{\beta} \text { as } z \rightarrow+\infty, \\
E_{\beta}(z) \rightarrow \frac{e^{z}}{\beta} \text { as } z \rightarrow+\infty .
\end{gathered}
$$

If $a(t)=a$ is a constant, then $u(t) \leq a E_{\beta}(\theta t), 0 \leq t \leq T$.

Applying Lemma 6 in Inequality (30), we obtain the validity of the following estimate:

$$
\begin{gathered}
y(t) \leq M_{1} y(0)+\frac{M_{2} c_{0}^{*} t^{\alpha}}{\alpha}+ \\
\left\lceil c b_{1} M_{2} \Gamma(\alpha)\right\rceil^{\frac{1}{\alpha}} \int_{0}^{t} E_{\alpha, 1}\left(\left\lceil c b_{1} M_{2} \Gamma(\alpha)\right\rceil^{\frac{1}{\alpha}}(t-s)^{\alpha}\right)\left(M_{1} y(0)+\frac{M_{2} c_{0}^{*} s^{\alpha}}{\alpha}\right) d s, 0 \leq t \leq T .
\end{gathered}
$$

Taking into account the notation, we obtain the first a priori estimate from here:

$$
\begin{gathered}
\left\|u_{m}\right\|_{2, \Omega}^{2}+\left\|\nabla u_{m}\right\|_{2, \Omega}^{2}+\int_{\Gamma} K(x, t)\left|u_{m}\right|^{\sigma} d \Gamma \\
\leq\left(M_{1}\left(1+\left\|u_{m}(x, 0)\right\|_{2, \Omega}^{2}+\left\|\nabla u_{m}(x, 0)\right\|_{2, \Omega}^{2}\right)+\frac{M_{2} c_{0}^{*} t^{\alpha}}{\alpha}\right) \\
+\left[c b_{1} M_{2} \Gamma(\alpha)\right]^{\frac{1}{\alpha}} \int_{0}^{t} E_{\alpha, 1}\left(\left[c b_{1} M_{2} \Gamma(\alpha)\right]^{\frac{1}{\alpha}}(t-s)^{\alpha}\right) \\
\times\left(M_{1}\left(1+\left\|u_{m}(x, 0)\right\|_{2, \Omega}^{2}+\left\|\nabla u_{m}(x, 0)\right\|_{2, \Omega}^{2}\right)+\frac{M_{2} c_{0}^{*} s^{\alpha}}{\alpha}\right) d s \leq C_{3}, 0 \leq t \leq T<\infty,
\end{gathered}
$$

where $C_{3}$ is a constant independent from $m \in N$.

We derive the second a priori estimate in the same way as for $2<p<\frac{2 n}{n-2}, n \geq 3$. Let us evaluate each term of the right part of Relation (17), applying the inequalities of Jung, Helder, and Lemma 5, we obtain:

$$
\begin{gathered}
\left|-\int_{\Omega} \nabla u_{m} D_{0, t}^{\alpha} \nabla u_{m} d x\right| \leq \frac{1}{2}\left\|D_{0, t}^{\alpha} \nabla u_{m}\right\|_{2, \Omega}^{2}+\frac{1}{2}\left\|\nabla u_{m}\right\|_{2, \Omega}^{2} \\
\left.\left.\left|-\int_{\Gamma} K(x, t)\right| u_{m}\right|^{\sigma-2} u_{m} D_{0, t}^{\alpha} u_{m} d \Gamma\left|\leq K_{1} \int_{\Gamma}\right| D_{0, t}^{\alpha} u_{m}|| u_{m}\right|^{\sigma-1} d \Gamma \\
\leq K_{1}\left(\int_{\Gamma}\left|u_{m}\right|^{\frac{2(n-1)(\sigma-1)}{n}} d \Gamma\right)^{\frac{n}{2(n-1)}}\left(\int_{\Gamma}\left|D_{0, t}^{\alpha} u_{m}\right|^{\frac{2(n-1)}{n-2}} d \Gamma\right)^{\frac{n-2}{2(n-1)}} \\
\leq K_{1} C_{1}^{\frac{\sigma-1}{\sigma}} C_{3}^{\sigma-1}\left(\left\|D_{0, t}^{\alpha} u_{m}\right\|_{2, \Omega}^{2}+\left\|\nabla D_{0, t}^{\alpha} u_{m}\right\|_{2, \Omega}^{2}\right)^{\frac{1}{2}} \\
\leq K_{1}^{2} C_{1}^{\frac{2(\sigma-1)}{\sigma}} C_{3}^{2(\sigma-1)}+\frac{1}{4}\left(\left\|D_{0, t}^{\alpha} u_{m}\right\|_{2, \Omega}^{2}+\left\|\nabla D_{0, t}^{\alpha} u_{m}\right\|_{2, \Omega}^{2}\right),
\end{gathered}
$$


where $2<\sigma<\frac{2(n-1)}{n-2}, n \geq 3$.

$$
\begin{gathered}
\left.\left.\left|\int_{\Omega} b(x, t)\right| u_{m}\right|^{p-2} u_{m} D_{0, t}^{\alpha} u_{m} d x\left|\leq b_{1} \int_{\Omega}\right| u_{m}\right|^{p-1} D_{0, t}^{\alpha} u_{m} d x \\
\leq b_{1}\left(\int_{\Omega}\left|u_{m}\right|^{2(p-1)} d x\right)^{\frac{1}{2}}\left(\int_{\Omega}\left|D_{0, t}^{\alpha} u_{m}\right|^{2} d x\right)^{\frac{1}{2}} \\
\leq b_{1}\left\|u_{m}\right\|_{2, \Omega}\left\|D_{0, t}^{\alpha} u_{m}\right\|_{2, \Omega} \leq b_{1} C_{1}^{\frac{1}{2}}\left\|D_{0, t}^{\alpha} u_{m}\right\|_{2, \Omega} \leq b_{1}^{2} C_{1}+\frac{1}{4}\left\|D_{0, t}^{\alpha} u_{m}\right\|_{2, \Omega},
\end{gathered}
$$

where $1<p \leq 2, n \geq 3$.

Substituting these inequalities into Identity (17), we obtain the following:

$$
\left\|D_{0, t}^{\alpha} u_{m}\right\|_{2, \Omega}^{2}+\left\|D_{0, t}^{\alpha} u_{m}\right\|_{2, \Omega}^{2} \leq C_{4},
$$

for all $t \in[0, T]$.

As before, multiplying Equality (7) by $d_{j}(t) \in C[0, T]$ and summing up both parts of the resulting identity over $\mathrm{j}=\overline{1, m}$, and by virtue of the above arguments, we obtain (23) almost everywhere $t \in[0, T]$.

Hence, as in the proof of Theorem 1, we obtain a proof of Theorem 3.

2.5. The Uniqueness of the Global Generalized Solution of the Problem K (1)-(3) for $1<p \leq 2$

Doing the same as in the case of $2<p<\frac{2 n}{n-2}, n \geq 3$, we prove the validity of the following theorem:

Theorem 4. Assume that $1<p \leq 2, n \geq 3$. Then for any function $u_{0}(x) \in W_{2}^{1}(\Omega)$, on interval $(0, T)$ there exists a unique generalized solution of the Problem K (1)-(3).

Proof of Theorem 4. Acting the same as in the proof of Theorem 2, we assume that the Problem $\mathrm{K}$ has two solutions $u_{1}(x, t)$ and $u_{2}(x, t)$. Then for the difference $u(x, t)=$ $u_{1}(x, t)-u_{2}(x, t)$ we obtain Identity (24), for any function $\omega(x, t) \in L_{2}\left(0, T ; W_{2}^{1}(\Omega)\right)$. Since $\omega(x, t) \in L_{2}\left(0, T ; W_{2}^{1}(\Omega)\right)$, assuming $\omega(x, t)=u(x, t)$, we have (25).

Then, applying the following known inequalities:

$$
\begin{gathered}
\left|\left(\left|u_{1}\right|_{q} u_{1}-\left|u_{2}\right|_{q} u_{2}\right)\left(u_{1}-u_{2}\right)\right| \geq\left|u_{1}-u_{2}\right|_{q+2}, q>0, \\
\left(\left|u_{1}\right|^{p-2} u_{1}-\left|u_{2}\right|^{p-2} u_{2}, u_{1}-u_{2}\right) \leq \gamma(p)\left|u_{1}-u_{2}\right|^{p}, 1<p \leq 2 .
\end{gathered}
$$

we estimate the right-hand side of (25); taking into account Inequality (31), we have:

$$
\left|\int_{\Omega} b(x, \tau)\left(\left|u_{1}\right|^{p-2} u_{1}-\left|u_{2}\right|^{p-2} u_{2}\right) u d x\right| \leq b_{1} \gamma(p)\|u\|_{p, \Omega}^{p} \leq c b_{1} \gamma(p)\left(1+\|u\|_{2, \Omega}^{2}+\|\nabla u\|_{2, \Omega}^{2}\right) \text {. }
$$

By virtue of (32) and Lemma 1, then from (25) we obtain:

$$
\begin{aligned}
& D_{0, t}^{\alpha} \int_{\Omega}\left[|u|^{2}+|\nabla u|^{2}\right] d x+C_{0} \int_{\Omega}\left[|u|^{2}+|\nabla u|^{2}\right] d x \\
+ & K_{0} \int_{\Gamma}|u|^{\sigma} d \Gamma \leq 2 c b_{1} \gamma(p)\left(1+\|u\|_{2, \Omega}^{2}+\|\nabla u\|_{2, \Omega}^{2}\right),
\end{aligned}
$$

where $C_{0}=\min \left\{2 c_{0}^{*} ; 2\right\}$.

Let us represent Inequality (33) in the form:

$$
\begin{gathered}
D_{0, t}^{\alpha}\left(1+\|u\|_{2, \Omega}^{2}+\|\nabla u\|_{2, \Omega}^{2}\right)+C_{0}\left(1+\|u\|_{2, \Omega}^{2}+\|\nabla u\|_{2, \Omega}^{2}\right) \\
\leq C_{0}+2 c b_{1} \gamma(p)\left(1+\|u\|_{2, \Omega}^{2}+\|\nabla u\|_{2, \Omega}^{2}\right) .
\end{gathered}
$$


or

$$
\begin{gathered}
D_{0, t}^{\alpha}\left(1+\|u\|_{2, \Omega}^{2}+\|\nabla u\|_{2, \Omega}^{2}\right)+C_{0}\left(1+\|u\|_{2, \Omega}^{2}+\|\nabla u\|_{2, \Omega}^{2}\right) \\
\leq\left(C_{0}+2 c b_{1} \gamma(p)\right)\left(1+\|u\|_{2, \Omega}^{2}+\|\nabla u\|_{2, \Omega}^{2}\right) .
\end{gathered}
$$
written as:

Denoting $y(t) \equiv 1+\left\|u_{m}(x, t)\right\|_{2, \Omega}^{2}+\left\|\nabla u_{m}(x, t)\right\|_{2, \Omega}^{2}$, then the last inequality will be

$$
D_{0, t}^{\alpha} y(t)+C_{0} y(t) \leq\left(2 c b_{1} \gamma(p)+C_{0}\right) y(t)
$$

From here, we obtain:

$$
y(t) \leq E_{\alpha, 1}\left(-C_{0} t^{\alpha}\right) y(0)+\left(C_{0}+2 c b_{1} \gamma(p)\right) \int_{0}^{t}(t-s)^{\alpha-1} E_{\alpha, \alpha}\left(-C_{0}(t-s)^{\alpha}\right) y(s) d s,
$$

In Inequality (34), applying (13) and (14), we obtain:

$$
y(t) \leq M_{1} y(0)+\left(C_{0}+2 c b_{1} \gamma(p)\right) M_{2} \int_{0}^{t}(t-s)^{\alpha-1} y(s) d s .
$$

From the latter, taking into account the above notation, we have:

$$
\begin{gathered}
1+\left\|u_{m}\right\|_{2, \Omega}^{2}+\left\|\nabla u_{m}\right\|_{2, \Omega}^{2} \leq M_{1}\left(1+\left\|u_{m}(x, 0)\right\|_{2, \Omega}^{2}+\left\|\nabla u_{m}(x, 0)\right\|_{2, \Omega}^{2}\right) \\
+C_{1} M_{2} \int_{0}^{t}(t-s)^{\alpha-1}\left(1+\left\|u_{m}(x, s)\right\|_{2, \Omega}^{2}+\left\|\nabla u_{m}(x, s)\right\|_{2, \Omega}^{2}\right) .
\end{gathered}
$$

We consider the function:

$$
U(t) \equiv \sup _{s \in[0, t]}\left(\left\|u_{m}(x, s)\right\|_{2, \Omega}^{2}+\left\|\nabla u_{m}(x, s)\right\|_{2, \Omega}^{2}\right)
$$

Then from Inequality (35) we obtain the following inequality:

$$
1+U(t) \leq M_{1}+C_{1} M_{2} \frac{t^{\alpha}}{\alpha}+C_{1} M_{2} \frac{t^{\alpha}}{\alpha} U(t) .
$$

It is clear that at $M_{1}+C_{1} M_{2} \frac{t^{\alpha}}{\alpha}<1, M_{1}<1, C_{1} M_{2} \frac{t^{\alpha}}{\alpha}<1$, i.e., $t<t_{1}, M_{1}<1$, where $t_{1}^{\alpha}=\min \left\{\frac{\alpha}{C_{1} M_{2}} ; \frac{\alpha\left(1-M_{1}\right)}{C_{1} M_{2}}\right\}$, Inequality (36) holds if and only if $U(t)=0$ at $t \leq t_{1}$. However, then, from (35), we obtain such an inequality:

$$
1+U\left(t_{2}\right) \leq M_{1}+C_{1} M_{2} \frac{t_{2}^{\alpha}-t_{1}^{\alpha}}{\alpha}+C_{1} M_{2} \frac{t_{2}^{\alpha}-t_{1}^{\alpha}}{\alpha} U\left(t_{2}\right),
$$

that under conditions

$$
M_{1}+C_{1} M_{2} \frac{t_{2}^{\alpha}-t_{1}^{\alpha}}{\alpha}<1, C_{1} M_{2} \frac{t_{2}^{\alpha}-t_{1}^{\alpha}}{\alpha}<1 \text {, i.e., } t_{2}^{\alpha}<2 t_{1}^{\alpha},
$$

holds if and only if $U(t)=0$ at $t \leq t_{2}$. Continuing this procedure, we will eventually obtain:

$$
U(t)=0 \text { for } t \in[0, T]
$$

which leads to $\int_{\Omega}\left(|u|^{2}+|\nabla u|^{2}\right) d x=0$ almost everywhere on the time interval, meaning that the weak generalized solution is unique. Thus, the uniqueness of the generalized solution of the problem $\mathrm{K}(1)-(3)$ is proved. 
2.6. Solvability of a Problem with Nonlinear Boundary Conditions for One Variant of a Fractional Order Pseudo-Parabolic Equation

As before, we consider in the cylinder $Q_{T}=\left\{(x, t): x \in \Omega, \Omega \subset R^{n}, 0<t<T\right\}$ the initial-boundary problem for Equation (1), assuming that the coefficient $b(x, t)=-q(x, t)$ :

$$
D_{0, t}^{\alpha}(u-\Delta u)-\Delta u+c(x, t) u+q(x, t)|u|^{p-2} u=0,
$$

where $q(x, t)$ is a given function. With respect to the coefficients of Equation (37), we assume that the following conditions are met:

$$
\begin{gathered}
c(x, t) \geq c_{0}^{*}>0,0<q_{0} \leq q(x, t) \leq q_{1}, \forall(x, t) \in Q_{T}, \\
\left|D_{0, t}^{\alpha} q(x, t)\right| \leq q_{2}, 0<K_{0} \leq K(x, t) \leq K_{1}, \forall(x, t) \in Q_{T} .
\end{gathered}
$$

Problem $\mathbf{K}_{\mathbf{q}}$. Find a solution of Equation (37) satisfying the conditions (2)-(3).

Similarly, as shown in Section 2, taking into account $b(x, t)=-q(x, t)$, the concept of a weak generalized solution of the problem $\mathrm{K}_{\mathrm{q}}$ is defined.

Theorem 5. Let Condition (38) be satisfied and $1 \leq p \leq \frac{2 n}{n-2}, 1 \leq \sigma \leq \frac{2(n-1)}{n-2}, n \geq 3$. Then for any function $u_{0}(x) \in W_{2}^{1}(\Omega)$ almost everywhere on the interval $(0, T)$ there is a weak generalized solution $u(x, t)$ of the Problem $K_{q}$.

Proof of Theorem 5. Proceeding in the same way as shown in Section 3, we will look for an approximate solution of the Problem $\mathrm{K}_{\mathrm{q}}$, in the form (6). In this case, $v_{m k}(t)$ will be found from the following system:

$$
\begin{aligned}
& \sum_{k=1}^{m} D_{0, t}^{\alpha} v_{m k}(t) \int_{\Omega}\left\{\Psi_{k} \Psi_{j}+\sum_{i=1}^{m} \frac{\partial \Psi_{k}}{\partial x_{i}} \frac{\partial \Psi_{j}}{\partial x_{i}}\right\} d x+\int_{\Omega} c(x, t) u_{m} \Psi_{j} d x+\int_{\Omega} \nabla u_{m} \nabla \Psi_{j} d x \\
& +\sum_{k=1}^{m} v_{m k} \int_{\Gamma} K(x, t)\left|u_{m}\right|^{\sigma-2} \Psi_{k} \Psi_{j} d \Gamma+\sum_{k=1}^{m} v_{m k} \int_{\Omega} q(x, t)\left|u_{m}\right|^{p-2} \Psi_{k} \Psi_{j} d x=0,
\end{aligned}
$$

with Conditions (8) and (9).

Let us introduce the notation

$$
\begin{gathered}
\vec{v}_{m}=\left\{v_{1 m}(t), \ldots, v_{m m}(t)\right\}^{T}, \vec{\alpha} \equiv\left\{\alpha_{1}, \ldots, \alpha_{m}\right\}^{T}, a_{k j}=\int_{\Omega}\left[\Psi_{k} \Psi_{j}+\left(\nabla \Psi_{\mathrm{k}} \nabla \Psi_{\mathrm{j}}\right)\right] d x, \\
C_{k j}=\int_{\Omega} c(x, t) \Psi_{k} \Psi_{j} d x+\int_{\Omega} \nabla \Psi_{k} \nabla \Psi_{j} d x, \\
q_{k j}=-\int_{\Gamma} K(x, t)\left|u_{m}\right|^{\sigma-2} \Psi_{k} \Psi_{j} d \Gamma-\int_{\Omega} q(x, t)\left|u_{m}\right|^{p-2} \Psi_{k} \Psi_{j} d x, \\
A_{m} \equiv\left\{a_{j k}\right\}, \vec{C}_{m} \equiv\left\{C_{j k}\right\}, \vec{F}_{m}\left(\vec{v}_{m}\right) \equiv\left\{q_{j k}\left(\vec{v}_{m}\right)\right\} \vec{v}_{m} .
\end{gathered}
$$

Then, the system of Equations (8) and (39) takes the matrix form:

$$
A_{m} D_{0, t}^{\alpha} \vec{v}_{m}+\vec{C}_{m} \vec{v}_{m}=-\vec{F}_{m}\left(\vec{v}_{m}\right), \vec{v}_{m}(0)=\vec{\alpha}
$$

Multiply both parts of (39) by $v_{m j}(t)$ and sum over $\mathrm{j}=\overline{1, m}$. As a result, we obtain:

$$
\begin{gathered}
\int_{\Omega}\left\{u_{m} D_{0, t}^{\alpha} u_{m} d x+\nabla u_{m} D_{0, t}^{\alpha} \nabla u_{m}\right\} d x+\int_{\Omega}\left|\nabla u_{m}\right|^{2} d x+\int_{\Omega} c(x, t)\left|u_{m}\right|^{2} d x \\
+\int_{\Gamma} K(x, t)\left|u_{m}\right|^{\sigma} d \Gamma+\int_{\Omega} q(x, t)\left|u_{m}\right|^{p} d x=0 .
\end{gathered}
$$

Applying Lemma 1 and Condition (38), we obtain the inequality:

$$
\begin{gathered}
\frac{1}{2} D_{0, t}^{\alpha} \int_{\Omega}\left\{\left|u_{m}\right|^{2}+\left|\nabla u_{m}\right|^{2}\right\} d x+\int_{\Omega}\left|\nabla u_{m}\right|^{2} d x+c_{0}^{*} \int_{\Omega}\left|u_{m}\right|^{2} d x \\
+K_{0} \int_{\Gamma}\left|u_{m}\right|^{\sigma} d \Gamma+q_{0} \int_{\Omega}\left|u_{m}\right|^{p} d x \leq 0 .
\end{gathered}
$$


From Inequality (40), it follows that

$$
\begin{gathered}
D_{0, t}^{\alpha} y(t)+C_{0} y(t) \leq 0, \\
y(0)=y_{0}=\left\|u_{m}(x, 0 \|)_{2, \Omega}^{2}+\right\| \nabla u_{m}(x, 0 \|)_{2, \Omega}^{2}
\end{gathered}
$$

where $C_{0}=\min \left\{2 c_{0}^{*} ; 2\right\}, y(t)=\left\|u_{m}(x, t \|)_{2, \Omega}^{2}+\right\| \nabla u_{m}(x, t \|)_{2, \Omega}^{2}$.

Now let $\bar{y}(t)$ be a solution of the following Cauchy problem:

$$
\begin{gathered}
D_{0, t}^{\alpha} \bar{y}(t)+C_{0} \bar{y}(t)=0, \\
\bar{y}(0)=\bar{y}_{0}=\left\|u_{m}(x, 0)\right\|_{2, \Omega}^{2}+\left\|\nabla u_{m}(x, 0)\right\|_{2, \Omega}^{2} .
\end{gathered}
$$

The solution of this problem can be written explicitly as:

$$
\bar{y}(t)=E_{\alpha, 1}\left(-C_{0} t^{\alpha}\right) y(0) \text {. }
$$

Since the Inequality $y(t) \leq \bar{y}(t)$ holds, and taking into account (13), we obtain:

$$
y(t) \leq E_{\alpha, 1}\left(-C_{0} t^{\alpha}\right) y(0) \leq \frac{M_{1} y(0)}{1+C_{0} t^{\alpha}} .
$$

Thus, it is shown that

$$
\left\|u_{m}(x, t)\right\|_{2, \Omega}^{2}+\left\|\nabla u_{m}(x, t)\right\|_{2, \Omega}^{2} \leq \frac{M_{1}\left(\|u(x, 0)\|_{2, \Omega}^{2}+\|\nabla u(x, 0)\|_{2, \Omega}^{2}\right)}{1+C_{0} t^{\alpha}} \leq C_{1}, \forall t \geq 0 .
$$

Now we multiply Equality (39) by $D_{0, t}^{\alpha} v_{m j}(t)$ and sum by $j=\overline{1, m}$. As a result, we obtain:

$$
\begin{gathered}
\left\|D_{0, t}^{\alpha} u_{m}\right\|_{2, \Omega}^{2}+\left\|\nabla D_{0, t}^{\alpha} u_{m}\right\|_{2, \Omega}^{2}= \\
-\int_{\Omega} \nabla u_{m} D_{0, t}^{\alpha} \nabla u_{m} d x-\int_{\Omega} c(x, t) u_{m} D_{0, t}^{\alpha} u_{m} d x \\
-\int_{\Gamma} K(x, t)\left|u_{m}\right|^{\sigma-2} u_{m} D_{0, t}^{\alpha} u_{m} d \Gamma-\int_{\Omega} q(x, t)\left|u_{m}\right|^{p-2} u_{m} D_{0, t}^{\alpha} u_{m} d x
\end{gathered}
$$

The right-hand side of Identity (41) is evaluated similarly as (17). Thus, from Identity (41) we obtain:

$$
\left\|D_{0, t}^{\alpha} u_{m}\right\|_{2, \Omega}^{2}+\left\|D_{0, t}^{\alpha} u_{m}\right\|_{2, \Omega}^{2} \leq C_{4},
$$

for all $t \in(0, T)$.

Furthermore, the proof of Theorem 5 is performed in the same way as when proving Theorem 3.

Now we prove the uniqueness of the weak generalized solution of the Problem $\mathrm{K}_{\mathrm{q}}$.

Theorem 6. Let $u_{0}(x) \in W_{2}^{1}(\Omega), p>1, n \geq 3$ be executed. Then a weak generalized solution of the Problem $K_{q}(37),(2)$, (3) on interval $(0, T)$ is unique.

Proof of Theorem 6. Assume that the Problem $\mathrm{K}_{\mathrm{q}}$ (37), (2), (3) has two solutions $u_{1}(x, t)$ and $u_{2}(x, t)$. Then their difference $u(x, t)=u_{1}(x, t)-u_{2}(x, t)$ satisfies the condition $u(x, 0)=0$ and the identity:

$$
\begin{gathered}
\int_{\Omega}\left(D_{0, t}^{\alpha} u \cdot \omega+\sum_{i=1}^{n} D_{0, t}^{\alpha} \frac{\partial u}{\partial x_{i}} \cdot \frac{\partial \omega}{\partial x_{i}}+c(x, t) u \omega\right) d x+\int_{\Gamma} K(x, t)\left(\left|u_{1}\right|^{\sigma-2} u_{1}-\left|u_{2}\right|^{\sigma-2} u_{2}\right) \omega d \Gamma \\
+\int_{\Omega} q(x, t)\left(\left|u_{1}\right|^{p-2} u_{1}-\left|u_{2}\right|^{p-2} u_{2}\right) \omega d x=0,
\end{gathered}
$$


Due to the fact that $\omega(x, t) \in L_{2}\left(0, T ; W_{2}^{1}(\Omega)\right)$, then as $\omega(x, t)$ we can take $u(x, t)$; i.e., suppose $\omega(x, t)=u(x, t)$ :

$$
\begin{gathered}
\int_{\Omega}\left(D_{0, t}^{\alpha} u \cdot u+\sum_{i=1}^{n} D_{0, t}^{\alpha} \frac{\partial u}{\partial x_{i}} \cdot \frac{\partial u}{\partial x_{i}}+c(x, t)|u|^{2}\right) d x+\int_{\Gamma} K(x, t)|u|^{\sigma-2}\left(\left|u_{1}\right|^{\sigma-2} u_{1}-\left|u_{2}\right|^{\sigma-2} u_{2}\right) u d \Gamma \\
+\int_{\Omega} q(x, t)\left(\left|u_{1}\right|^{p-2} u_{1}-\left|u_{2}\right|^{p-2} u_{2}\right) u d x=0 .
\end{gathered}
$$

By applying Lemma 1 to Inequality (31), from (42), we obtain the differential inequality:

$$
D_{0, t}^{\alpha} \int_{\Omega}\left[|u|^{2}+|\nabla u|^{2}\right] d x+C_{0} \int_{\Omega}\left[|u|^{2}+|\nabla u|^{2}\right] d x+K_{0} \int_{\Gamma}|u|^{\sigma} d \Gamma+q_{0} \int_{\Omega}|u|^{p} d x \leq 0,
$$

where $C_{0}=\min \left\{2 c_{0}^{*} ; 2\right\}$.

Inequality (43) can be written as:

$$
D_{0, t}^{\alpha} \int_{\Omega}\left[|u|^{2}+|\nabla u|^{2}\right] d x+C_{0} \int_{\Omega}\left[|u|^{2}+|\nabla u|^{2}\right] d x \leq 0 .
$$

From the last inequality it follows that

$$
\begin{gathered}
D_{0, t}^{\alpha} y(t)+C_{0} y(t) \leq 0, \\
y(0)=\left\|u_{m}(x, 0)\right\|_{2, \Omega}^{2}+\left\|\nabla u_{m}(x, 0)\right\|_{2, \Omega}^{2}=0,
\end{gathered}
$$

where:

$$
y(t) \equiv\left\|u_{m}(x, t)\right\|_{2, \Omega}^{2}+\left\|\nabla u_{m}(x, t)\right\|_{2, \Omega}^{2} .
$$

Now let $\bar{y}(t)$ be a solution of the following Cauchy problem:

$$
\begin{gathered}
D_{0, t}^{\alpha} \bar{y}(t)+C_{0} \bar{y}(t)=0, \\
\bar{y}(0)=\left\|u_{m}(x, 0)\right\|_{2, \Omega}^{2}+\left\|\nabla u_{m}(x, 0)\right\|_{2, \Omega}^{2}=0 .
\end{gathered}
$$

The solution of this problem can be written explicitly as follows:

$$
\bar{y}(t)=E_{\alpha, 1}\left(-C_{0} t^{\alpha}\right) y(0) \text {. }
$$

Since the inequality $y(t) \leq \bar{y}(t)$ is satisfied by virtue of Estimate (13), we obtain:

$$
y(t) \leq E_{\alpha, 1}\left(-C_{0} t^{\alpha}\right) y(0), t \geq 0,
$$

which, by virtue of (44), implies the uniqueness of a weak generalized solution of the problem $\mathrm{K}_{\mathrm{q}}(37)$, (2), (3). Thus, Theorem 6 is proved.

\section{Discussion and Conclusions}

Note that the problems of solvability of the Dirichlet and Neumann problem analogues for a linear degenerate pseudo-parabolic equation with a fractional Caputo derivative are studied in [3,6-8].

For integer values $\alpha=1$ and $\alpha=2$, the theorems on the existence and uniqueness of a weak solution of the analogue of the problem K (1)-(3) are proved in [30-32].

This paper provides the first study of the problems of unique solvability of initial boundary value problems for a quasi-linear pseudo-parabolic fractional equation with nonlinear boundary conditions with Caputo fractional differentiation operators.

The following main results are obtained for the fractional order quasi-linear pseudoparabolic Equation (1):

- The questions of unique solvability of boundary value problems are formulated and investigated, the difference of which is that the fractional differentiation operators 
participate both in the equation itself and in the boundary condition in the form of a nonlinear condition;

- A theorem on the local solvability of the problem $\mathrm{K}$ is proved for $2<p<\frac{2 n}{2}$, $2<\sigma<\frac{2(n-1)}{n-2}, n \geq 3$;

- The uniqueness theorem of the local solution of the problem $\mathrm{K}$ is proved if the conditions are satisfied $\left(2<p<\frac{2 n}{2}, n \geq 3\right)$;

- The uniqueness theorem of a global solution of the Problem K for $1<p \leq 2$ and the existence theorem of a global solution of the problem $\mathrm{K}$ under the conditions $1<\sigma<\frac{2(n-1)}{n-2}, n \geq 3$ are established;

- For one variant of a pseudo-parabolic equation of fractional order, the existence and uniqueness theorems of a global solution of an initial boundary value problem with nonlinear boundary conditions are proved.

Author Contributions: Problem statement and methodology, A.S.B. and S.E.A.; original draft preparation and full realization, S.E.A. and K.S.B. All authors have read and agreed to the published version of the manuscript.

Funding: The work was carried out with the financial support of the Ministry of Education and Science of the Republic of Kazakhstan under grant No. AP08857604.

Institutional Review Board Statement: Not applicable.

Informed Consent Statement: Not applicable.

Data Availability Statement: Not applicable.

Acknowledgments: The authors are thankful to the editor and anonymous referees for their valuable comments and suggestions.

Conflicts of Interest: The authors declare no conflict of interest.

\section{References}

1. Showalter, R.E.; Ting, T.W. Pseudoparabolic partial differential equations. SIAM J. Math. Anal. 1970, 1, 1-26. [CrossRef]

2. Beshtokov, M.K. Boundary value problems for a pseudoparabolic equation with the Caputo fractional derivative. Transl. Differ. Uravn 2019, 55, 884-893. [CrossRef]

3. Tuan, N.A.; Regan, D.O.; Baleanu, D.; Tuan, N.H. On time fractional pseudo-parabolic equations with nonlocal integral conditions. Evol. Equ. Control Theory 2020. [CrossRef]

4. Sousa, C.V.J.; de Oliveira, C.E. Fractional order pseudoparabolic partial differential equation: Ulam-Hyers stability. Bull. Braz. Math. Soc. New Ser. 2019, 50, 393-420. [CrossRef]

5. Korpusov, M.O.; Sveshnikov, A.G. Blow-up of solutions of a Sobolev-type equation with a nonlocal source. Sib. Math. J. 2005, 46, 567-578. [CrossRef]

6. Bouziani, A. Solvability of nonlinear pseudoparabolic equation with a nonlocal boundary condition. Nonlinear Anal. 2003, 55, 883-904. [CrossRef]

7. Su, R.; Xu, J. Global existence and finite time blow-up for a class of semilinear pseudo-parabolic equations. J. Funct. Anal. 2013, 264, 2732-2763. [CrossRef]

8. Nakhushev, A.M. Fractional Calculus and Its Application; Fizmatlit: Moscow, Russia, 2003; p. 272. ISBN 5-9221-0440-3. (In Russian)

9. Podlubny, I. Fractional Differential Equations; Academic Press: London, UK, 1999; p. 368. ISBN 0125588402.

10. Samko, S.G.; Kilbas, A.A.; Marichev, O.I. Fractional Integrals and Derivatives, Theory and Applications; Gordon and Breach: Yverdon, Switzerland, 1993; p. 976. ISBN 2-8-8124-864-0.

11. Kilbas, A.A.; Srivastava, H.M.; Trujillo, J.J. Theory and Applications of Fractional Differential Equations; Elsevier: Amsterdam, The Netherlands, 2006; Volume 204, pp. 325-348. ISBN 9780444518323.

12. Wang, $\mathrm{X} . ; \mathrm{Xu}, \mathrm{R}$. Global existence and finite time blowup for a nonlocal semilinear pseudo-parabolic equation. Adv. Nonlinear Anal. 2021, 10, 261-288. [CrossRef]

13. Gopala Rao, V.R.; Ting, T.W. Solutions of pseudo-heat equations in the whole space. Arch. Ration. Mech. Anal. 1972, 49, 57-78. [CrossRef]

14. Brill, H. A Semilinear Sobolev evolution equation in a Banach space. J. Differ. Equ. 1977, 24, 412-425. [CrossRef]

15. Berdyshev, A.; Cabada, A.; Karimov, E. On the existence of eigenvalues of a boundary value problem with transmitting condition of the integral form for a parabolic-hyperbolic equation. Mathematics 2020, 8, 1030. [CrossRef]

16. Beshtokov, M.K. To boundary-value problems for degenerating pseudoparabolic equations with Gerasimov-Caputo fractional derivative. Izv. Vyssh. Uchebn. Zaved. Mater. 2018, 10, 3-16. [CrossRef] 
17. Beshtokov, M.K. Boundary-value problems for loaded pseudoparabolic equations of fractional order and difference methods of their solving. Russ. Math. 2019, 63, 1-10. [CrossRef]

18. Binh, H.D.; Hoang, L.N.; Baleanu, D.; Van Ho, T.K. Continuity Result on the Order of a Nonlinear Fractional Pseudo-Parabolic Equation with Caputo Derivative. Fractal Fract. 2021, 5, 41. [CrossRef]

19. Tuan, N.H.; Huynh, L.N.; Ngoc, T.B.; Zhou, Y. On a backward problem for nonlinear fractional diffusion equations. Appl. Math. Lett. 2018, 92, 76-84. [CrossRef]

20. Ngoc, T.B.; Zhou, Y.; O’Regan, D.; Tuan, N.H. On a terminal value problem for pseudoparabolic equations involving RiemannLiouville fractional derivatives. Appl. Math. Lett. 2020, 106, 106373. [CrossRef]

21. Abbas, S.; Benchohra, M. Upper and lower solutions method for impulsive partial hyperbolic differential equations with fractional order. Nonlinear Anal. Hybrid. Syst. 2010, 4, 406-413. [CrossRef]

22. Srivastava, H.M.; Fernandez, A.; Baleanu, D. Some new fractional-calculus connections between Mittag-Leffler functions. Mathematics 2019, 7, 485. [CrossRef]

23. Alikhanov, A.A. A Priory Estimates for Solutions of Boundary Value Problems for Fractional-Order Equations. Differ. Equ. 2010, 46, 658-664. (In Russian) [CrossRef]

24. Butler, G.; Rogers, T. A Generalization of Lemma of Bihari and Applications to Pointwise Estimates for Integral Equations. J. Math. Anal. Appl. 1971, 33, 77-81. [CrossRef]

25. Ladyzhenskaia, O.A.; Solonnikov, V.A.; Uraltseva, N.N. Linear and Quasi-Linear Equations of Parabolic Type; Translations of Mathematical Monographs; American Mathematical Society: Providence, RI, USA, 1968; p. 648.

26. Taukenova, F.I.; Shkhanukov-Lafishev, M.K. Difference methods for solving boundary value problems for fractional differential equations. Comput. Math. Math. Phys. 2006, 46, 1785-1795. [CrossRef]

27. Kochubei, A.N. Diffusion of fractional order. Differ. Equ. 1990, 26, 485-492.

28. Zhou, Y. Basic Theory of Fractional Differential Equations. Nonlinear Anal. Theory Methods Appl. 2014, 69, 2677-2682.

29. Seki, K.; Wojcik, M.; Tachiya, M. Fractional reaction-diffusion equation. J. Chem. Phys. 2003, 119, 2165-2170. [CrossRef]

30. Berdyshev, A.S.; Aitzhanov, S.E.; Zhumagul, G.O. Solvability of Pseudoparabolic Equations with Non-linear boundary Condition. Lobachevskii J. Math. 2020, 41, 772-1783. [CrossRef]

31. Makarov, P.A. Destruction of the solution of the initial boundary value problem for the generalized Boussinesq equation with a nonlinear boundary condition. Math. Notes 2012, 92, 567-582. [CrossRef]

32. Alshin, A.B.; Korpusov, M.O.; Sveshnikov, A.G. Blow-Up in Nonlinear Sobolev Type; Walter de Gruyter Co.: Berlin, Germany, 2011; p. xii+648. ISBN 978-3-11-025527-0. [CrossRef]

33. Henry, D. Geometric Theory of Semilinear Parabolic Equations; Springer: New York, NY, USA, 1981; p. 353. ISBN 978-3-540-10557-2. 